\title{
Plasma velocity in hydromagnetic dynamos
}

Manuel Núñez

Citation: Journal of Mathematical Physics 43, 3202 (2002); doi: 10.1063/1.1473679

View online: https://doi.org/10.1063/1.1473679

View Table of Contents: http://aip.scitation.org/toc/jmp/43/6

Published by the American Institute of Physics

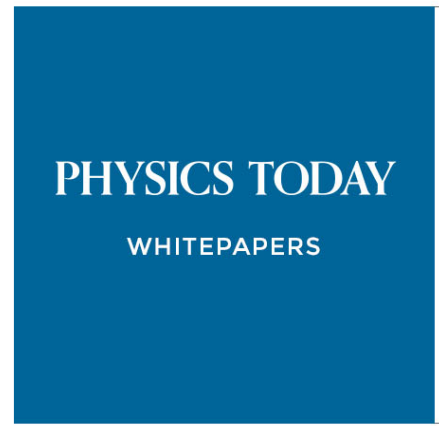

MANAGER'S GUIDE

Accelerate R\&D with

Multiphysics Simulation

\section{READ NOW}

PRESENTED BY $\checkmark \subset \mathrm{MSOL}$ 


\section{Plasma velocity in hydromagnetic dynamos}

Manuel Núñez ${ }^{\text {a) }}$

Departamento de Análisis Matemático, Universidad de Valladolid, 47005 Valladolid, Spain

(Received 12 February 2002; accepted for publication 25 February 2002)

Hydromagnetic dynamos are plasma configurations generating for some time an exponentially increasing magnetic field. By using a number of functional inequalities, we estimate the rate of increase of magnetic energy in terms of the plasma resistivity and diferent norms on the plasma velocity. Our bounds are proved to be optimal as far as the powers of the relevant magnitudes are concerned. (C) 2002 American Institute of Physics. [DOI: 10.1063/1.1473679]

\section{INTRODUCTION}

A hydromagnetic dynamo in a plasma is a configuration allowing for a finite time an exponential growth of the magnetic field. The behavior of the main magnitudes in an incompressible plasma is governed by the magnetohydrodynamic (MHD) system: the velocity $\mathbf{u}$, magnetic field $\mathbf{B}$, kinetic pressure $p$, viscosity $\nu$ and resistivity $\eta$ satisfy, after the usual normalizations,

$$
\begin{gathered}
\frac{\partial \mathbf{u}}{\partial t}=\nu \Delta \mathbf{u}-\mathbf{u} \cdot \nabla \mathbf{u}+\mathbf{B} \cdot \nabla \mathbf{B}-\nabla p-\nabla\left(\frac{B^{2}}{2}\right), \\
\frac{\partial \mathbf{B}}{\partial t}=\eta \Delta \mathbf{B}-\mathbf{u} \cdot \nabla \mathbf{B}+\mathbf{B} \cdot \nabla \mathbf{u}, \\
\nabla \cdot \mathbf{u}=\nabla \cdot \mathbf{B}=0 .
\end{gathered}
$$

The MHD system, for any boundary conditions allowing no input of energy from the outside, is dissipative (see e.g. Ref. 1). Therefore, any growth of magnetic energy must ultimately be done at the expense of the kinetic one, i.e., of the plasma velocity. Once this velocity is taken for granted, the magnetic field is governed by the induction equation (2), and the magnetic energy by the integral identity obtained making the scalar product of (2) and $\mathbf{B}$ :

$$
\frac{1}{2} \frac{\partial}{\partial t} \int_{\Omega} B^{2} d V=\eta \int_{\Omega} \Delta \mathbf{B} \cdot \mathbf{B} d V+\int_{\Omega} \mathbf{B} \cdot \nabla \mathbf{u} \cdot \mathbf{B} d V-\int_{\Omega} \mathbf{u} \cdot \nabla \mathbf{B} \cdot \mathbf{B} d V .
$$

If we assume $\left.\mathbf{u} \cdot \mathbf{n}\right|_{\partial \Omega}=0$ (i.e., the fluid does not cross the boundary), the last integral vanishes. As for the term

$$
\eta \int_{\Omega} \Delta \mathbf{B} \cdot \mathbf{B} d V=-\eta \int_{\Omega}|\nabla \mathbf{B}|^{2} d V+\frac{\eta}{2} \int_{\partial \Omega} \frac{\partial B^{2}}{\partial n} d \sigma
$$

provided there is no input of magnetic energy from the outside,

$$
\int_{\partial \Omega} \frac{\partial B^{2}}{\partial n} d \sigma \leqslant 0
$$

yields the fundamental energy inequality

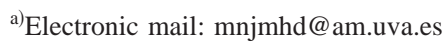




$$
\frac{1}{2} \frac{\partial}{\partial t} \int_{\Omega} B^{2} d V \leqslant-\eta \int_{\Omega}|\nabla \mathbf{B}|^{2} d V+\int_{\Omega} \mathbf{B} \cdot \nabla \mathbf{u} \cdot \mathbf{B} d V
$$

Condition (5) holds (with an equality) for Dirichlet $\left(\left.\mathbf{B}\right|_{\partial \Omega}=\mathbf{0}\right)$ or perfect conductor $\left(\left.\mathbf{B} \cdot \mathbf{n}\right|_{\partial \Omega}\right.$ $\left.=0 ;(\nabla \times \mathbf{B}) \times\left.\mathbf{n}\right|_{\partial \Omega}=\mathbf{0}\right)$ conditions. We will assume either periodic boundary conditions in a box $\Omega$ with

$$
\int_{\Omega} \mathbf{u} d V=\int_{\Omega} \mathbf{B} d V=\mathbf{0}
$$

or

$$
\left.\mathbf{u} \cdot \mathbf{n}\right|_{\partial \Omega}=\left.\mathbf{B} \cdot \mathbf{n}\right|_{\partial \Omega}=0,
$$

in a smooth $N$-dimensional domain $\Omega$. Thus we will take (6) as the starting inequality. The first term on the right-hand side of (6) accounts for the diffusive effects of the resistivity, while the second is an advective term showing the transport of the magnetic field by the flow. In fact, in ideal plasmas $(\eta=0)$ the magnetic field lines are transported by the plasma as material points and the field strength may be enhanced by this process.

From here one may ignore the diffusive term and bound the advective one by

$$
\left|\int_{\Omega} \mathbf{B} \cdot \nabla \mathbf{u} \cdot \mathbf{B} d V\right| \leqslant \frac{1}{2}\left\|\nabla \mathbf{u}+(\nabla \mathbf{u})^{t}\right\|_{\infty} \int_{\Omega} B^{2} d V
$$

where \|\|$_{\infty}$ means the maximum norm and ( $)^{t}$ the transposed matrix (see, e.g., Ref. 2). Therefore, the growth parameter $\gamma$ satisfies

$$
\gamma \leqslant \frac{1}{2}\left\|\nabla \mathbf{u}+(\nabla \mathbf{u})^{t}\right\|_{\infty} .
$$

This estimate goes back to Backus. ${ }^{3}$ Thus the maximal exponential growth rate does not exceed the largest eigenvalue of the strain matrix $\frac{1}{2}\left(\nabla \mathbf{u}+(\nabla \mathbf{u})^{t}\right)$. This elementary inequality has some merits: the main one is that it does not depend on the resistivity and therefore it holds even when $\eta \rightarrow 0$. A velocity configuration yielding a dynamo even when $\eta \rightarrow 0\left[\inf _{\eta \rightarrow 0} \gamma(\eta)>0\right]$ is called a fast dynamo $;{ }^{4}$ this is an extensively studied subject. On the minus side, we first note that anything involving the gradient of the velocity is somewhat unsatisfactory. This is so because in many turbulent flows there exist sharp changes in the velocity vector, whereas the velocity size remains moderate. Indeed, on general principles one may reject an extremely large plasma velocity, but there is no physical reason to exclude rapid variations of the flow: thus any norm on the velocity itself may be much smaller than the norm of the gradient. Moreover, the maximum norm is the worst possible: it could happen that the plasma remains almost quiescent except for a tiny portion which alone ensures that the maximum of the strain matrix is large. One does not expect the magnetic energy of the whole domain to be governed by a minute portion of the plasma. We will see that (9) may be significantly improved.

\section{THE MAIN ESTIMATES}

Certain subspaces of the Sobolev space $H^{1}(\Omega)$ possess the property that

$$
\|f\|_{H^{1}} \leqslant k\|\nabla f\|_{2},
$$

i.e., the $L^{2}$-norm of $f$ is dominated by the norm of its gradient. These are the so-called Poincare inequalities. One of the most general descriptions of spaces where one of these inequalities holds is as follows (see Refs. 1 and 5): let $p$ be a continuous seminorm [i.e., a continuous norm, except for the fact that $p(f)=0$ does not imply $f=0]$ on $H^{1}(\Omega)$ such that for every constant function 
$g \neq 0, p(g) \neq 0$. Then any subspace $H$ of $H^{1}(\Omega)$, such that $p(f)=0$ for all $f \in H$, satisfies a Poincaré inequality. Among the many examples of such seminorms, we will use the following ones:

$$
\begin{gathered}
p(\mathbf{f})=\left|\int_{\Omega} \mathbf{f} d V\right|, \\
p(\mathbf{f})=\int_{\partial \Omega}|\mathbf{f} \cdot \mathbf{n}| d \sigma .
\end{gathered}
$$

Equation (11) covers periodic problems because of the zero mean condition (7), while (12) covers the remaining cases, since (8) holds. That the seminorm $p$ of (12) is continuous follows from the fact that the trace of any function $f \in H^{1}(\Omega)$ at the boundary belongs to $L^{1}(\partial \Omega)$ [and even to $\left.L^{2}(\partial \Omega)\right]$.

Our main tool will be a weak version of the Gagliardo-Nirenberg inequality (Ref. 6, pp. 65-68): denoting as usual by \|\|$_{p}$ the norm in $L^{p}(\Omega)$,

$$
\|f\|_{p} \leqslant C\|f\|_{H^{1}}^{\tau}\|f\|_{2}^{1-\tau}
$$

where $C$ is a constant depending only on the domain, $\tau=(N / 2)-(N / p)$. This holds provided $p$ $\geqslant 2,(N / 2)-(N / p)<1$, i.e., $p<2 N /(N-2)$. Thus, for $N=3,2 \leqslant p<6$; for $N=2$, any $p \geqslant 2$ is admissible.

Since

$$
\int_{\Omega} \mathbf{B} \cdot \nabla \mathbf{u} \cdot \mathbf{B} d V=-\int_{\Omega} \mathbf{B} \cdot \nabla \mathbf{B} \cdot \mathbf{u} d V
$$

by the inequality of Cauchy-Schwarz

$$
\left|\int_{\Omega} \mathbf{B} \cdot \nabla \mathbf{u} \cdot \mathbf{B} d V\right| \leqslant \int_{\Omega}|\mathbf{B}\|\nabla \mathbf{B}\| \mathbf{u}| d V \leqslant\|\mathbf{B}\|_{p}\|\nabla \mathbf{B}\|_{2}\|\mathbf{u}\|_{q},
$$

for any positive $p, q$ such that $1 / p+1 / q=\frac{1}{2}$; hence $p, q \geqslant 2$. By (13),

$$
\|\mathbf{B}\|_{p} \leqslant C\|\mathbf{B}\|_{H^{1}}^{(N / 2)-(N / p)}\|\mathbf{B}\|_{2}^{1-(N / 2)+(N / p)}=C\|\mathbf{B}\|_{H^{1}}^{N / q}\|\mathbf{B}\|_{2}^{1-(N / q)},
$$

provided $p<2 N /(N-2)$, i.e., $q>N$. Thus,

$$
\left|\int_{\Omega} \mathbf{B} \cdot \nabla \mathbf{u} \cdot \mathbf{B} d V\right| \leqslant C\|\mathbf{B}\|_{H^{1}}^{N / q}\|\mathbf{B}\|_{2}^{1-(N / q)}\|\nabla \mathbf{B}\|_{2}\|\mathbf{u}\|_{q} .
$$

Let us use now the Poincaré inequality, written as

$$
\|\mathbf{B}\|_{H^{1}} \leqslant k\|\nabla \mathbf{B}\|_{2} .
$$

We have

$$
\left|\int_{\Omega} \mathbf{B} \cdot \nabla \mathbf{u} \cdot \mathbf{B} d V\right| \leqslant C k^{N / q}\|\nabla \mathbf{B}\|_{2}^{1+(N / q)}\|\mathbf{B}\|_{2}^{1-(N / q)}\|\mathbf{u}\|_{q} \cdot
$$

Let us denote

$$
r=\frac{1}{2}-\frac{N}{2 q}
$$


We may write the right-hand term as

$$
C k^{N / q}\left(\|\nabla \mathbf{B}\|_{2}^{2}\right)^{1-r}\left(\|\mathbf{B}\|_{2}^{2}\right)^{r}\|\mathbf{u}\|_{q}=\left(\alpha\|\nabla \mathbf{B}\|_{2}^{2}\right)^{1-r}\left(\left(C k^{N / q}\|\mathbf{u}\|_{q}\right)^{1 / r} \alpha^{-(1-r) / r}\|\mathbf{B}\|_{2}^{2}\right)^{r},
$$

where $\alpha$ is a positive constant to be determined later. By using the classical inequality

$$
x^{r} y^{1-r} \leqslant r x+(1-r) y
$$

for $x, y>0$ (which amounts to the convexity of the exponential function), we find

$$
\left|\int_{\Omega} \mathbf{B} \cdot \nabla \mathbf{u} \cdot \mathbf{B} d V\right| \leqslant(1-r) \alpha\|\nabla \mathbf{B}\|_{2}^{2}+r C^{1 / r} k^{N / q r} \alpha^{-(1-r) / r}\|\mathbf{u}\|_{q}^{1 / r}\|\mathbf{B}\|_{2}^{2} .
$$

Take now $\alpha=\eta /(1-r)$. Then the term in $\nabla \mathbf{B}$ cancels with the dissipative term in (6), and we are left with

$$
\frac{1}{2} \frac{d}{d t}\|\mathbf{B}\|_{2}^{2} \leqslant r(1-r)^{(1-r) / r}\left(C k^{N / q} \eta^{-(1-r)}\|\mathbf{u}\|_{q}\right)^{1 / r}\|\mathbf{B}\|_{2}^{2}
$$

Therefore, if there exists a magnetic dynamo of exponential growth rate $\gamma$, for any $q>N$,

$$
\gamma \leqslant 2 r(1-r)^{(1-r) / r}\left(C k^{N / q} \eta^{-(1-r)}\|\mathbf{u}\|_{q}\right)^{1 / r},
$$

where $r$ is given by (19). $C$ and $k$ are universal constants. The estimate improves with large $q$ and $\eta$, and becomes singular as $\eta \rightarrow 0$ or $r \rightarrow 0$ (i.e., $q \rightarrow N$ ). For $q \rightarrow \infty$ it becomes

$$
\gamma \leqslant \frac{1}{2} C \eta^{-1}\|\mathbf{u}\|_{\infty}^{2}
$$

which improves the Backus bound (10) in the sense that it does not need the velocity gradient, although the resistivity occurs. The estimate (23) is satisfactory in the sense that it involves an integral norm of the velocity and therefore it is a measure of its mean size: it shows that the dynamo cannot be governed by what happens in small regions of the plasma, although these may be relevant in the process of stretching which is basic in the dynamo process. However, the physically most important norm of the velocity is the kinetic energy $\|\mathbf{u}\|_{2}$, which is not reached by (23). For $N=2$ it lies at the lower limit and the constants blow there; for $N=3$ it is well beyond reach. To see that this is a physical fact and not merely the result of poor bounds, we will prove that (23) is a sharp inequality as concerns the order of the magnitudes.

\section{COUNTEREXAMPLES FOR LOWER ORDER NORMS}

We will consider an initial condition formed by velocity and field depending only on the radius, and radially directed. Then $\mathbf{B} \cdot \nabla \mathbf{u}$ is also radially directed and the term $\mathbf{B} \cdot \nabla \mathbf{u} \cdot \mathbf{B}$ is precisely $|\mathbf{B}|^{2}|\nabla \mathbf{u}|$. Specifically, assume $\mathbf{B}=B(R) \mathbf{e}_{r}, \mathbf{e}_{r}$ the unit radial vector, $B$ decreasing linearly from $B=h$ at $r=0$ to $B=0$ at $r=L: B(r)=h-h r / L$ for $r \in[0, L], B(r)=0$ for $r>L$. Take $\mathbf{u}$ $=\mathbf{B}$. These magnitudes are not really smooth, as they fail to be differentiable at $r=0$ and $r$ $=L$, but they can be uniformly approximated by smooth functions such that the values of all the integrals tend to the respective values for our chosen functions.

First, since the Jacobian in dimension $N$ depends on $r$ like $r^{N-1}$, the norm $\|\mathbf{u}\|_{q}$ behaves like $h L^{N / q}$, and $\|\mathbf{B}\|_{2}$ like $h L^{N / 2} .|\nabla \mathbf{u}|$ is identical to $h / L$ for $r \in[0, L]$, and zero otherwise; thus

$$
\int_{\Omega}|\mathbf{B}|^{2}|\nabla \mathbf{u}| d V=h L^{-1} \int_{\Omega}|\mathbf{B}|^{2} d V \sim h L^{-1} h^{2} L^{N}=h^{3} L^{N-1} .
$$

On the other hand, 


$$
\eta \int_{\Omega}|\nabla \mathbf{B}|^{2} d V \sim \eta h^{2} L^{N-2},
$$

so that the behavior of the right-hand side of Eq. (6), as a function of $h$ and $L$, is

$$
-\eta h^{2} L^{N-2}+h^{3} L^{N-1} \text {. }
$$

Therefore, any exponential growth rate $\gamma$ should be of the order of (27) divided by $h^{2} L^{N}$, i.e.,

$$
\gamma \sim-\eta L^{-2}+h L^{-1}
$$

while $\|\mathbf{u}\|_{q} \sim h L^{N / q}$.

Assume $q<N$, and take $s$ such that $1<s<N / q$. Choose $h=L^{-s}$. Then, for $L$ small,

$$
\gamma \sim-\eta L^{-2}+L^{-1-s} \sim L^{-1-s} .
$$

While $\|\mathbf{u}\|_{q} \sim L^{N / q-s}$ tends to zero with $L, \gamma \rightarrow \infty$. Thus there is no possible bound of $\gamma$ in terms of $\|\mathbf{u}\|_{q}$.

For $q=N$, we must avoid the possible indetermination in (29) occurring for $h=L^{-1}$. Therefore, we take $h=L^{-1} \log L^{-1}$. For $L$ small enough,

$$
-\eta L^{-2}+L^{-2} \log L^{-1} \sim L^{-2} \log L^{-1},
$$

whereas $\|\mathbf{u}\|_{q} \sim \log L^{-1}$. Since obviously, for any power $n$,

$$
\left(\log L^{-1}\right)^{n} \ll L^{-2} \log L^{-1},
$$

when $L \rightarrow 0$, there cannot be any bound of $\gamma$ in terms of any power of $\|\mathbf{u}\|_{q}$.

Logically the method fails for $q>N$, because any attempt of setting $h=L^{-s}$ would yield a negative power of $L$ at both sides; we could choose an adequate power on the right-hand side to make the magnitudes comparable. Notice that our test functions are localized in a neigborhood of $\mathbf{0}$ and therefore satisfy our boundary conditions.

\section{CONCLUSIONS}

Defining hydromagnetic dynamos as plasma configurations producing an exponential growth of the magnetic field for some time, it is desirable to bound the possible growth rates in terms of the size of the plasma velocity. Classical inequalities involve the maximum norms of the velocity gradient, which are unsuitable for several reasons. We prove a bound of the growth rate by a power of the $L^{q}$-norm of the velocity and the conductivity, for any $q$ strictly larger than the space dimension $N$. The estimate blows up in the limit $q=N$ as well as in the ideal limit of zero resistivity. It is shown by examples that there cannot be analogous bounds for $q \leqslant N$.

\section{ACKNOWLEDGMENT}

This work was partially supported by the Ministry of Science of Spain under Grant No. BMF2000-0814.

\footnotetext{
${ }^{1}$ R. Temam, Infinite-Dimensional Dynamical Systems in Mechanics and Physics (Springer, New York, 1988).

${ }^{2}$ M. Núñez, J. Math. Phys. 38, 1583 (1997).

${ }^{3}$ G. Backus, Ann. Phys. (N.Y.) 4, 372 (1958).

${ }^{4}$ S. Childress and A. D. Gilbert, Stretch, Twist and Fold: the Fast Dynamo, (Springer, New York, 1995).

${ }^{5}$ J. Deny and J. L. Lions, Ann. Inst. Fourier 5, 305 (1954).

${ }^{6}$ V. G. Maz'ja, Sobolev Spaces (Springer, Berlin, 1985).
} 